\title{
Revisión del sistema de gestión de pavimentos de la red ciclorrutas de Bogotá
}

\section{Review of the pavement management system of the Bogotá bike-path network}

\author{
Gilberto Martínez Arguelles*1, Luis Guillermo Fuentes**, Lina María Torregroza Aldana*** \\ * Universidad de Piloto de Colombia, Bogotá D.C., COLOMBIA \\ ** Universidad del Norte, Barranquilla, COLOMBIA \\ ***Ingetec S.A., Bogotá D.C., COLOMBIA \\ Fecha de recepción: $28 / 10 / 2010$ \\ Fecha de aceptación: 30/ 03/2011 \\ Resumen \\ PAG. 150 - 170

\begin{abstract}
El documento presenta una revisión objetiva sobre el sistema de gestión de ciclorrutas de la ciudad de Bogotá. Los resultados de la revisión muestran la necesidad de establecer un manual de fallas para ciclorrutas, que contenga los deterioros reales en este tipo de estructuras y las causas generadoras. La predicción del deterioro de las ciclorrutas, requiere de la definición de tramos testigos que alimenten una base de datos que permita el desarrollo de modelos empíricos. El documento propone elementos claves que se deben complementar y que debe tener como mínimo un sistema de gestión de infraestructura, tales como; el desarrollo de modelos de deterioro, definición de estándares de mantenimiento y la evaluación social en la fase de priorización de intervenciones. Estos elementos deben ser considerados para estructurar una próxima versión del SGC. Los resultados del estudio muestran diferentes aspectos que se deben mejorar y desarrollar, como es el caso de curvas de deterioro para las ciclorrutas, el desarrollo de un índice de confort para usuarios de las ciclorrutas y la definición de una guía de intervenciones en función del estado del tramo.
\end{abstract}

Palabras Clave: Ciclorrutas, sistemas de gestión de pavimentos, mantenimiento, modelos de deterioro

Abstract

The paper presents a review of the Bogota bike-path network management system. The results of the study showed that it is necessary to establish a specific manual for bicycle paths with the potential damage in such structures. The prediction of the distress on the bike paths requires the definition of monitoring sections to feed a database that allows the development of empirical models. The document proposed key elements that must contain an infrastructure management system, such as: models for deterioration development, definition of maintenance standards and social assessment at the stage of prioritizing interventions. These factors should be considered to structure a future version of the bike-path management system. The results of the study showed different topic that must be improved and developed, such as; the definition of a comfort index to bike-path user and the necessity of establishing a maintenance guidebook.

Keywords: Bike-path, pavement management system, maintenance, deterioration models

\section{Introducción}

Las ciclorrutas en Bogotá fueron consideradas desde su concepción como elementos de espacio público de carácter recreativo, para esparcimiento y para propiciar la actividad física. El proyecto de Ciclorrutas fue propuesto dentro del Plan de desarrollo (PD) 1995 - 1997 “Formar Ciudad". Fue denominado bajo el nombre "red vial de ciclovías" y se considera como uno de los proyectos pertenecientes a la prioridad en Espacio Público. Su objetivo fue construir un sistema de ciclorrutas permanentes en el Distrito Capital que articulara el sistema hídrico y el sistema verde metropolitano y que sirviera,

\section{Introduction}

Bike-paths in Bogota were planned from a very beginning as elements for public spaces of recreational nature, intended for leisure and promotion of physical activity. The Bike-paths Project was proposed under the Development Project (DP) 1995 1997 "Formar Ciudad". It was called "red vial de ciclo vías" (Bike-path Network) and it is considered as one of the Public Space priority projects. Its purpose was to build up a standing bike-path network in the Capital District to organize the storm water drainage system and the green metropolitan system mainly serving,

\footnotetext{
1 Autor de correspondencia / Corresponding author:

E-mail: gilberto-martinez@unipiloto.edu.co
} 
principalmente, como medio de recreación (Instituto de Desarrollo Urbano de Bogotá ((IDU), 2010a). En contraste con lo anterior, para la siguiente administración del Distrito, el Plan de Desarrollo 1998 - 2001 “Por la Bogotá que Queremos", incluyó el componente ciclorrutas como elemento fundamental, otorgándole importancia como alternativa para mejorar la movilidad en la ciudad. El tema de las ciclorrutas tomó gran importancia desde entonces y es así como la inversión en estudios, diseños y construcción de la infraestructura de ciclorrutas ha ocupado un espacio primordial dentro de los planes de desarrollo de la última década, erigiendo a Bogotá como la capital suramericana con la red más grande de ciclorrutas con cerca de $344 \mathrm{~km}$ de longitud (IDU, 2010a).

Uno de los factores que permitió el desarrollo y crecimiento de la red de ciclorrutas en la capital fue el Plan Maestro de CicloRuta (PMC) (IDU, 2010b), definido como una estrategia orientada a promover la movilización cotidiana en bicicleta por la ciudad de Bogotá, con la finalidad de reducir el tráfico y lograr positivos dividendos sociales, económicos y ambientales.

El transporte en bicicleta a través de la red de ciclorrutas juega un papel fundamental en la movilidad de los bogotanos y la bicicleta se constituye como un potencial medio de transporte en los centros urbanos, principalmente para la realización de viajes cortos (IDU, 2010b). Por lo tanto, además de efectivas políticas que incentiven el uso de la bicicleta, resulta fundamental mantener la red de ciclorrutas en buenas condiciones, permitiendo un ágil y seguro desplazamiento de los usuarios entre su origen y su destino. La inversión de la infraestructura de las ciclorrutas es gigante, considerando el costo de una cinta de mezcla asfáltica de $0.05 \mathrm{~m}$ de espesor por $2 \mathrm{~m}$ de ancho y $344 \mathrm{~km}$ de longitud que tiene la red de ciclorrutas de Bogotá. Por esta razón, se constituye de carácter urgente la aplicación de políticas de conservación y de administración sobre la red de ciclorrutas de Bogotá. $\mathrm{Al}$ respecto, el IDU de Bogotá ha dispuesto de una metodología para determinar el Índice de Condición de la Ciclorruta - ICC (IDU y TNM Limited, 1999), descrito más adelante en el presente artículo.

Adicionalmente, en este documento se presenta una revisión sobre el sistema de ciclorrutas en el Distrito Capital desde la óptica de la Gestión de Infraestructura Vial, proponiendo elementos claves que deben ser incorporados al sistema de gestión de ciclorrutas con el objetivo de contribuir en el avance, hacia un sistema de administración sostenible de la infraestructura de las ciclorrutas. as a recreational network (Instituto de Desarrollo Urbano de Bogotá ((IDU), 2010a). On the other hand, the following District Administration`s Development Plan 1998 - 2001, "For Por la Bogotá que Queremos"- included the bikepath network as a core element to become an alternative to improve the mobility of the city. Bike-path topic became relevant ever since and therefore investment in studies, design and bike-path infrastructure construction have been carried out it the last decade, raising Bogota as the south American Capital city with the greatest bike-path network I of $344 \mathrm{~km}$ length (IDU, 2010a).

One of the factors that allowed the development and growth of bike-paths in the capital city was the BikePath Master Plan (PMC) (IDU, 2010b), defined as a strategy intended to promote daily transportation in Bogota city using bicycles, which purpose was to reduce traffic and to achieve positive social, economical and environmental dividends.

Transportation by means of bicycles alongside the bike-path network plays a fundamental role for Bogota's citizens, since they provide an alternative transportation means for the urban community, mainly for short range distances (IDU, 2010 b). Therefore, besides effective policies promoting the use of bicycles, it is fundamental to maintain the bike-path network in good conditions thus allowing an agile and safe ride to users from departure to arrival points. The investment in bikepath infrastructure is huge, considering the cost for an asphaltic mixture trip of $0.05 \mathrm{~m}$ thickness by $2 \mathrm{~m}$ width and $344 \mathrm{~km}$ length comprising Bogota's bike-path network. This is why the implementation of conservation policies and an adequate management system for Bogota's bike-path network becomes urgent. In this aspect, the IDU in Bogota has implemented a methodology to determine the Condition Index of the Bike-path Network - ICC (IDU y TNM Limited, 1999), further described in the current paper.

Additionally, this paper shows a review on the bike-path network in the Capital District from a Road Infrastructure Management point of view, proposing key elements to be incorporated to the bike-path network management system that will ensure its sustainability. 
Teniendo en cuenta que más de un $90 \%$ de la red de ciclorrutas está conformada por estructuras de pavimento tipo flexible, se presenta un análisis del mecanismo de deterioro mayormente observado y se formulan recomendaciones para mitigarlo.

\section{Descripción de la infraestructura de las ciclorutas en Bogotá}

La red de ciclorrutas de Bogotá está conformada por $344 \mathrm{~km}$ que se extienden a lo largo de 19 de las 20 localidades de la ciudad (se excluye la localidad de Sumapaz, localidad $100 \%$ rural). Por jerarquización, la red de ciclorrutas de Bogotá se divide en: red principal, red secundaria, red complementaria y un pequeño grupo conocido como ciclorrutas zonales (ver Tabla 1 y Figura 1).
Taking into account that more than $90 \%$ of bike-path network is composed of flexible type pavement structures, the authors present an analysis of the most common structural failures observed on the Bike-path Network and recommendations are proposed as mitigation actions.

\section{Description of bike-path infrastructure in Bogotá}

The bike-path network in Bogota comprises $344 \mathrm{~km}$ along 19 out of 20 communities of the city (Sumapaz location is excluded as it is $100 \%$ rural). According to hierarchy, Bogota Bike-Path network is divided into: Primary network, secondary network and a small group known as zone bike-paths (ref. Table 1 and Figure 1).

Tabla 1. Jerarquías de la red de ciclorrutas en Bogotá

Table 1. Bogota's bike-path network hierarchy

\begin{tabular}{||l|l||}
\hline Tipo de red/Network Type & Descripción/Description \\
\hline Primaria/Primary & $\begin{array}{l}\text { Es la red que une en forma más directa los polos de atracción, recogiendo además flujos de ciclistas de la red } \\
\text { secundaria / Main network directly connecting attraction poles, collects bikers'flows from secondary network }\end{array}$ \\
\hline Secundaria/Secondary & $\begin{array}{l}\text { Es la red alimentadora de la red principal, colecta y distribuye los flujos de ciclistas desde los centros de atracción } \\
\text { o centros de vivienda / Feeds the primary network, collects and distributes bikers' flows from attraction poles } \\
\text { and housing centers }\end{array}$ \\
\hline Complementaria/Complementary & $\begin{array}{l}\text { Enlazan y dan continuidad a la red / Link and provide continuity to the network } \\
\text { Configura el sistema de malla. Incluyen la red ambiental y recreativa, las redes locales y de barrio y el sistema } \\
\text { de parques lineales / Configures the external network. Includes environmental and recreational networks, local } \\
\text { and neighborhood networks and linear parks system }\end{array}$ \\
\hline Zonales/Zone & $\begin{array}{l}\text { Son parte integral del sistema vial y recreativo, a escala sectorial y de barrio / Constitute an integral part of road } \\
\text { and recreational system at sector and neighborhood scale }\end{array}$ \\
\hline
\end{tabular}

Nota: Adaptado de www.idu.gov.co/Note: Adapted from www.idu.gov.co

La Figura 1 muestra el esquema funcional propuesto por el PMC. En la imagen se describe cómo a través de los diferentes niveles de redes (principal, secundaria complementaria y zonal) se conectan los centros de vivienda con el centro económico de una ciudad, y a su vez, cómo se interconecta un centro de vivienda con sus similares en la ciudad.
Figure 1 shows a functional layout proposed by the PMC. The image describes how housing centers are connected to the economical city center by means of different network levels (primary, secondary, complementary and zone networks) and how a housing center is interconnected to similar networks in the city. 


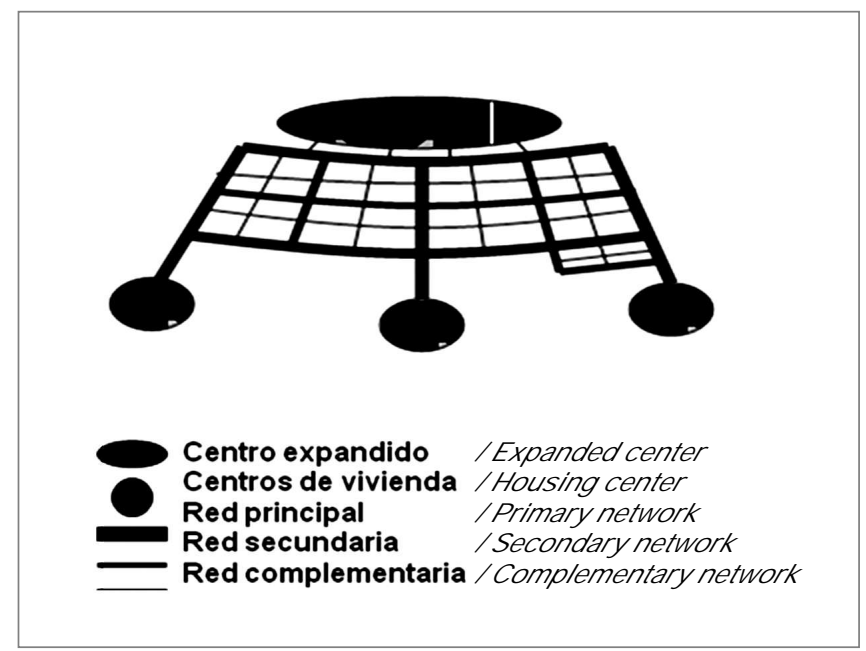

Figura 1. Esquema funcional de la red. Fuente: PMC

Figure 1. Network Functional Layout. Source PMC

Como toda red de transporte, su diseño debe obedecer al cumplimiento de unos parámetros mínimos de geometría y operación, lineamientos que se encuentran definidos en el Manual de Diseño de Ciclorrutas (MDC) elaborado dentro del marco de la consultoría para la elaboración del Plan Maestro de Ciclorrutas. La red está compuesta por una tipología de secciones variables ubicadas sobre andenes, rondas ambientales y separadores. EI MDC establece anchos de sección de ciclorrutas de $\mathbf{2 . 7 5} \mathbf{m}$ para tráfico menor a 1500 usuarios/día (sentido bidireccional) y $3.5 \mathrm{~m}$ para ciclorrutas con tráfico igual o superior a 1500 usuarios/día (sentido bidireccional), dejando siempre $0.5 \mathrm{~m}$ de holgura sobre el ancho efectivo (ver Ecuación 1).
Every transportation network must fulfill minimum geometry and operational parameters, which are defined on the Bike-path Design Guidelines (MDC) developed under an advisory framework for the implementation of Bike-path Master Plan. Such network comprises a variable section typology located on platforms, environmental courses and spacers. MDC establishes bike-paths section widths of $\mathbf{2 . 7 5} \mathbf{m}$ for traffic levels lower than 1500 users/day (both way) and $3.5 \mathrm{~m}$ for bike-paths for traffic levels equal or greater than 1500 users/day (both way), always considering $0.5 \mathrm{~m}$ clearance over the effective width (see Equation 1).

$$
\mathrm{AC}=\mathrm{Ae}+\mathrm{H}
$$

Donde:

AC: ancho de ciclorruta

Ae: ancho efectivo

$\mathrm{H}$ : holgura (EI MDC considera $0.5 \mathrm{~m}$ de holgura) El ancho mínimo efectivo de una pista unidireccional es de $2.25 \mathrm{~m}$.

Las Figuras 2 a la 4 muestran algunas secciones típicas de la red de ciclorrutas de la ciudad de Bogotá. Sin embargo el gran porcentaje de ciclorrutas en la ciudad presenta un ancho de $2 \mathrm{~m}$.
Where:

$A C$ : bike-path width

Ae: effective width

$H$ : clearance (MDC considers 0.5 clearance) The minimum width for a one way lane is $2.25 \mathrm{~m}$.

Figures from 2 to 4 show some typical bike-path sections in Bogota City. However, most bike-paths are 2 m width only. 


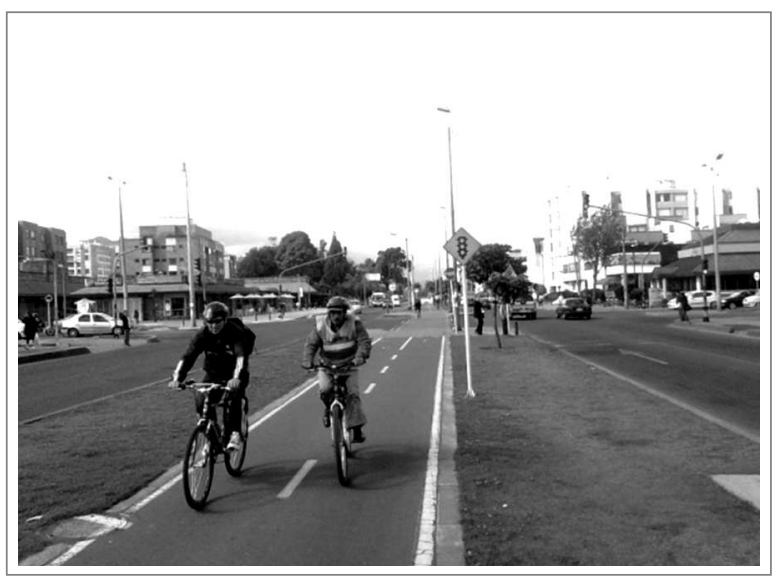

Figura 2. Sección de ciclo ruta en separador Figure 2. Bike-path section on spacer

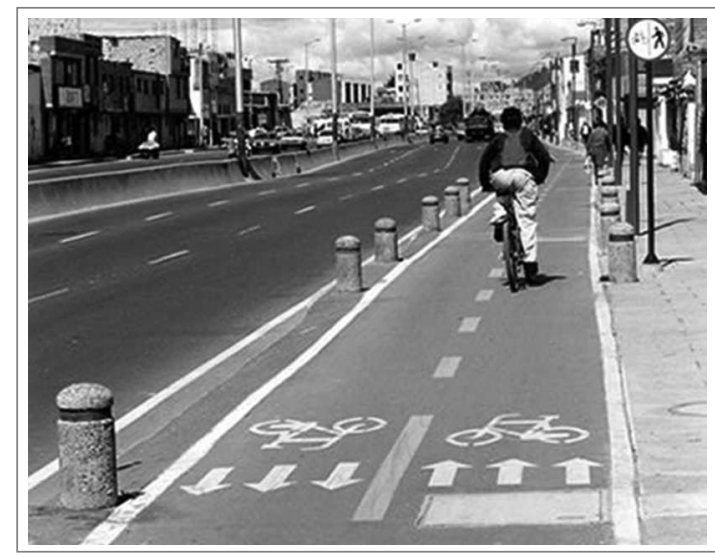

Figura 3. Sección de ciclorrutas sobre andén. Adaptado de ww.idu.gov.co Figure 3. Bike-path section on platform. Adapted from www.idu.gov.co.

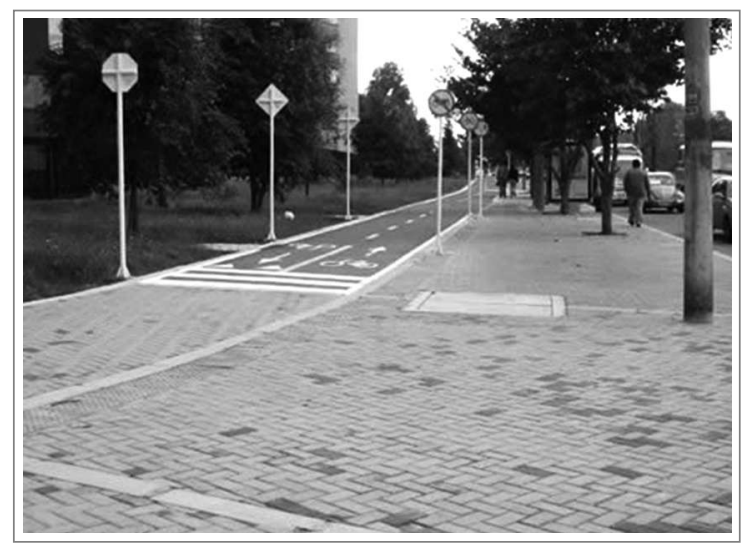

Figura 4. Sección de ciclorrutas sobre andén, superficie en adoquín y carpeta asfáltica. Adaptado de www.idu.gov.co Figure 4. Bike-path section on platform, paving stone surface and asphaltic layer. Adapted from www.idu.gov.co. 


\section{Sistemas de gestión de infraestructura}

Las ciclorrutas como cualquier elemento de la infraestructura de una ciudad, deben ser consideradas como parte del patrimonio de la misma. Por lo tanto, es primordial que las autoridades responsables prevengan, atiendan y corrijan de manera oportuna el deterioro que éstas sufran. Para que esto se pueda llevar a cabo de la mejor manera, es necesario desarrollar un Plan de Acción de Conservación, meta que se persigue cuando se utilizan los sistemas de gestión, entendido como una herramienta de decisión para aplicar las acciones más adecuadas, considerando su costo y estableciendo prioridades (De Solminihac, 2005, Haas et al., 1994).

Solminihac (2005) estableció como elementos básicos que configuran un sistema de gestión de infraestructura vial los siguientes:

- Información de inventario de la red

- Información del estado funcional y estructural de los elementos de la vía

- Modelos de predicción del comportamiento durante su vida útil

- Estándares de conservación para el deterioro actual y futuro

- Evaluación económica de la distintas alternativas de conservación y/o rehabilitación

- Configuración de un programa de actuación en el periodo de análisis

De los elementos antes mencionados, vale la pena resaltar que los factores de mayor complejidad para una agencia u organismo estatal son el desarrollo de los modelos de deterioro y la definición de los estándares de conservación para el deterioro actual y futuro. Para facilitar esta tarea a las agencias, el Banco Mundial ha desarrollado desde 1968 el modelo HDM-Highways Development Management en su versión III y IV (World Bank, 2000), herramienta técnica y económica de modelación de escenarios que permite mediante la utilización de un software, realizar simulaciones de una red o un determinado proyecto utilizando modelos de deterioros que vienen incluidos en el programa y que pueden ser calibrados para las condiciones económicas y geográficas de cualquier país.

De acuerdo con lo descrito por De Solminihac (2005), un sistema de gestión para el caso específico de una red de ciclorrutas debería incluir los seis aspectos mencionados, subrayando que tanto la definición de estándares de conservación, su evaluación económica y la configuración de un programa de actuación en un periodo de análisis, son herramientas que dependen de los recursos disponibles para el mantenimiento de la red,

\section{Infrastructure management system}

Bike-paths as any other city infrastructure element must be considered as an assets of the city. Therefore, the fundamental role of responsible authorities is to prevent, detect and correct in due course deterioration underwent by such bike-paths. In order to properly carry out such actions, it is necessary to create a Conservation Action Plan, purpose of any management system implementation, to be considered as a decision-making tool to implement the most adequate actions, considering costs involved and establishing priorities (De Solminihac, 2005, Haas et al., 1994).

Solminihac (2005) stated basic elements configuring the parameters of road infrastructure management:

- Information on network inventory.

- Information on functional and structural conditions of road elements.

- Predictive behavior models during operating life.

- Conservation standards for current and future deterioration.

- Economical evaluation on different conservation and/or rehabilitation alternatives.

- Action plan layout during analysis period.

From above elements, one should highlight that the most complex factors for an agency or state organization are the development and implementation of deterioration models and the elaboration of conservation standards for current and future deterioration. In order to facilitate such task, since 1968 the World Bank has developed the model HDM-Highways Development Management, version III and IV (World Bank, 2000), which is an economical and technical tool for scenario modelling, allowing by means of a software, to carry out simulations on a network or specific project by using deteriorarion models built-in the software, which can be calibrated for economical and geographical conditions in any country.

According to De Solmihihac (2005) statements, a management system for the specific case of a bike-path network should include the six above mentioned aspects, emphazising that conservation standards definition, economical evaluation and action program layout during analysis period are tools depending on available resouces for the network maintainance program, 
pero de igual forma de las políticas de mantenimiento definidas. En la Figura 5 se muestra una agrupación de los elementos antes mencionados en función de la dependencia o independencia de cada uno con respecto a las políticas coyunturales de un gobierno. Los tres aspectos iniciales: inventario de la red, información de estado funcional-estructural y los modelos de predicción del comportamiento durante su vida útil, son parámetros técnicos que dependen de las características propias de la red, de los materiales utilizados, de los atributos funcionales (rugosidad, fricción, sensación de confort) y de los modelos desarrollados en función de los tipos de materiales y durabilidad de los mismos. Por lo tanto, encontramos medidas que dependen de parámetros concretos y parámetros dependientes de la disponibilidad de recursos y de la acertada o desacertada política de conservación de una entidad frente a la red de estudio. and equally depending on defined maintenance policies. Figure 5 shows the above mentioned group of elements in function of dependance or independance regarding short-term policies established by a government. The first initial aspects: information on network inventory, information on functional and structural conditions and predictive behavior models during operating life are technical parameters that depend on the network own characteristics, on materials employed, on functional attributes (roughness, friction and comfort sensation) and on models developed in function of material types and their durability. Therefore, it is seen that these measures depend on specific parameters, parameters depending on resource availability and also depending on appropriate or inappropriate conservation policies settled by authorities in the studied network.
Parámetros independientes de las políticas de gobierno Parameters independent from governmental policies

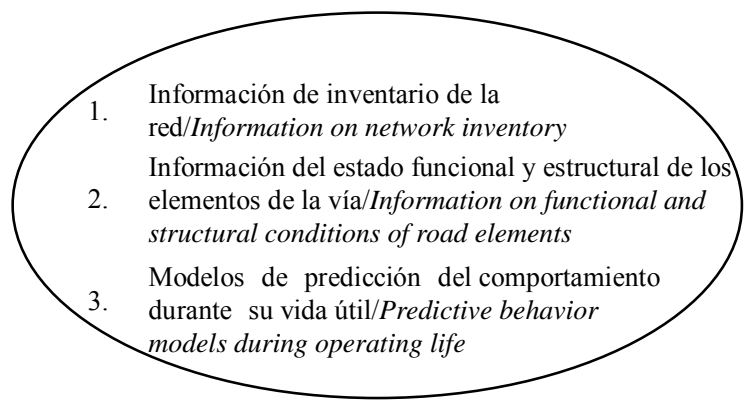

Parámetros dependientes de las políticas de gobierno Parameters depending on governmental policies

Figura 5. Dependencia de los elementos de un sistema de gestión de vial en las decisiones del gobierno

Figure 5. Elements depending on a road management system in a government decision making process

\section{Sistema de gestión actual de la red de ciclorrutas de Bogotá}

El sistema de gestión de las ciclorrutas en la ciudad de Bogotá está basado en la alimentación de la base de datos del IDU, mediante la elaboración de un inventario inicial de la infraestructura y la introducción periódica (como mínimo cada dos años se lleva a cabo el inventario) de parámetros indicadores del estado funcional y deterioro superficial (IDU y TNM Ltd (1999)).

\section{Current bike-path network management system in Bogotá}

The bike-path management system in Bogota is based on a data base feedback by IDU, by means of the elaboration of an initial infrastructure inventory and data periodically registered (inventory carried out every two years as minimum), including parameters indicating functional condition and surface deterioration (IDU and TNM Ltd. (1999)). 
El sistema de administración para Bogotá asigna a cada elemento de la infraestructura (andén, calzada de pavimento, separador y ciclorruta) un código de identificación, conocido como Código de Identificación Vial (CIV), el cual se encuentra georeferenciado dentro de la base de datos del IDU que puede ser consultada en una plataforma SIG (Sistema de información geográfica) como es mostrado en la Figura 6.
The Management System in Bogota assigns an identification code to each infrastructure element (platform, pavement carriage way, spacer and bike-path), which is known as Road Identification Code (CIV) that is georeferenced in IDU's data base and can be looked up from a GIS platform system (Geographical Information System), as shown in Figure 6.

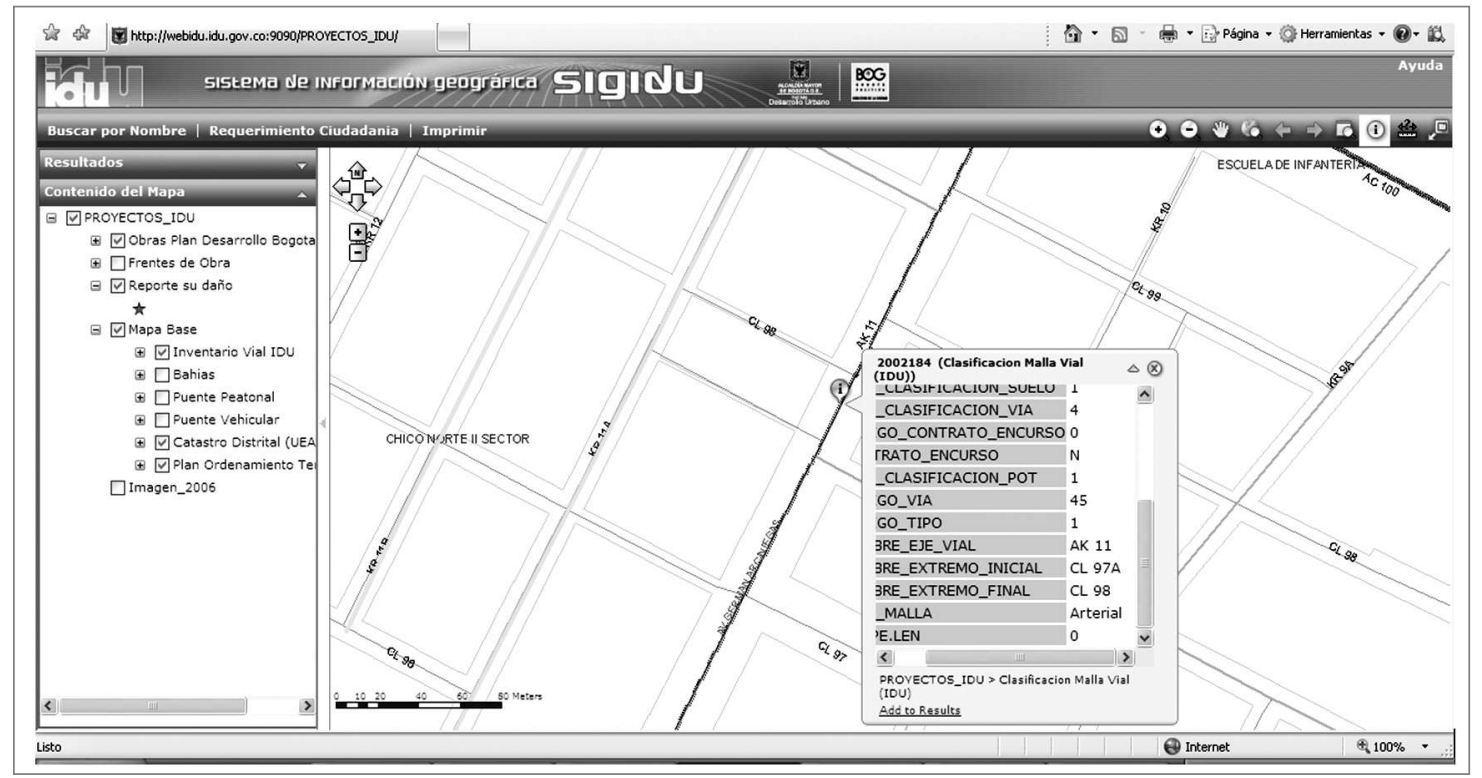

Figura 6. Sistema de georreferenciación IDU de la ciudad de Bogotá

Figure 6. IDU Geo-reference System in Bogotá

La Figura 7 muestra un esquema de identificación de las características geométricas de una cinta de ciclorrutas, como números de carriles, ancho, longitud y tipo de superficie.
Figure 7 shows an identification layout on the geometric characteristics of a pike-path belt, as well as number of lanes, width, length and surface type.

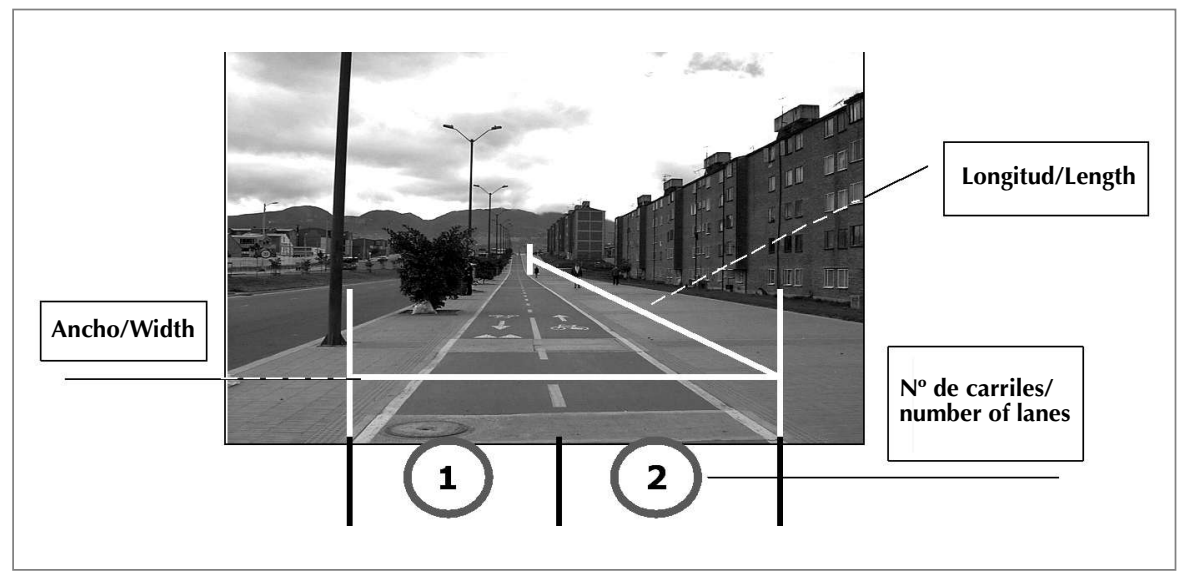

Figura 7. Ubicación y geometría de la ciclorruta. (IDU, 1999)

Figure 7. Location and geometry of bike-path (IDU, 1999) 
Los datos que se obtienen del inventario de la red de ciclorrutas son los siguientes:

- Índice de Rugosidad Internacional - IRI

- Tipo de Falla

- Severidad de la Falla

- Extensión de la Falla

- Tipo de superficie

- Geometría

Las fallas consideradas para la determinación del Índice Superficial de las Ciclorrutas se presentan en la Tabla 2. Estas fallas corresponden al mismo listado de fallas utilizado para la determinación del índice de estado de los pavimentos vehiculares. No obstante, es necesario aclarar que tanto las fallas como los mecanismos de deterioro de un pavimento vehicular son diferentes a las presentados en los pavimentos de una red de ciclorrutas. La literatura muestra trabajos realizados en la identificación de fallas sobre redes de ciclorrutas en donde se valoran además de defectos superficiales, condiciones de movilidad (geometría, manejabilidad) (Gharaibeh et al., 1998).
Data obtained from bike-path network inventory are the following:

- International Roughness Index - IRI

- Distress type

- Distress severity

- Distress extent

- Surface type

- Geometry

The distresses considered for the determination of Surface Bike-path Index are shown in Table 2. Such distresses are the same as those on the distress list used for determining vehicle pavement condition index. However, it is necessary to make clear that distress as well as deterioration mechanisms for vehicular pavement are quite different from failures in bike-path networks. Different studies have identified the failure conditions for bike-path networks focusing on superficial defects and mobility conditions (geometry and maneuverability) (Gharaibeh et al., 1998).

Tabla 2. Listado de fallas en ciclorrutas

Table 2. Bike-paths Distress List

\begin{tabular}{|c|c|c|c|c|c|}
\hline & Pavimentos Rígidos/Rigid Pavements & & $\begin{array}{l}\text { Pavimentos Flexibles/Flexible } \\
\text { Pavements }\end{array}$ & & $\begin{array}{l}\text { Pavimentos Articulados/ } \\
\text { Hinged Pavements }\end{array}$ \\
\hline Código de Falla & Descripción/Description & Código de Falla & Descripción/Description & Código de Falla & Descripción/Description \\
\hline 1 & Peladuras/Scabbing & 14 & Peladuras/Scabbing & \multirow[t]{2}{*}{25} & \multirow{2}{*}{$\begin{array}{l}\text { Deterioro sello de arena/ } \\
\text { sand sealing deterioration }\end{array}$} \\
\hline \multirow[t]{2}{*}{2} & \multirow{2}{*}{$\begin{array}{l}\text { Pulimiento de la superficie/Grinded } \\
\text { surface }\end{array}$} & 15 & Exudación/Bleeding & & \\
\hline & & \multirow[t]{2}{*}{16} & \multirow{2}{*}{$\begin{array}{l}\text { Baches descubiertos/non-covered } \\
\text { potholes }\end{array}$} & \multirow[t]{3}{*}{26} & \multirow{3}{*}{$\begin{array}{l}\text { Desintegración del } \\
\text { adoquin/stone pavement } \\
\text { decay }\end{array}$} \\
\hline 3 & Descascaramiento fisuras & & & & \\
\hline & capilares/Capillary peeling cracking & \multirow[t]{2}{*}{17} & \multirow{2}{*}{$\begin{array}{l}\text { Desintegración de bordes/edges } \\
\text { decay }\end{array}$} & & \\
\hline \multirow[t]{2}{*}{4} & \multirow{2}{*}{$\begin{array}{l}\text { Bache-Desintegración total/Pothole } \\
\text { - total decay }\end{array}$} & & & 27 & Desunión/De-bonding \\
\hline & & 18 & Hundimientos/soil sinking & 28 & Huecos/Holes \\
\hline \multirow[t]{2}{*}{5} & \multirow{2}{*}{$\begin{array}{l}\text { Hundimiento-depresión/Soil sinking } \\
\text { - depression }\end{array}$} & \multirow[t]{2}{*}{19} & \multirow{2}{*}{$\begin{array}{l}\text { Corrugaciones y Desplazamiento/ } \\
\text { ribbing and deflection }\end{array}$} & 29 & Fisuración/Cracking \\
\hline & & & & \multirow[t]{2}{*}{30} & \multirow[t]{2}{*}{ Escalonamiento/Stepping } \\
\hline 6 & Dislocamiento/Mis-matching & \multirow[t]{2}{*}{20} & \multirow{2}{*}{$\begin{array}{l}\text { Fisuramiento Longitudinal/ } \\
\text { Longitudinal cracking }\end{array}$} & & \\
\hline 7 & Fisura Longitudinal/Longitudinal & & & \multirow[t]{10}{*}{31} & \multirow{10}{*}{$\begin{array}{l}\text { Ondulaciones y } \\
\text { Hundimientos/soil rippling } \\
\text { and soil sinking }\end{array}$} \\
\hline & crack & \multirow[t]{2}{*}{21} & \multirow{2}{*}{$\begin{array}{l}\text { Fisuramiento Transversal/Cross-over } \\
\text { cracking }\end{array}$} & & \\
\hline \multirow[t]{2}{*}{8} & \multirow{2}{*}{$\begin{array}{l}\text { Fisuramiento Transversal/Cross-over } \\
\text { cracking }\end{array}$} & & & & \\
\hline & & \multirow[t]{2}{*}{22} & \multirow{2}{*}{$\begin{array}{l}\text { Fisuramiento de borde/border } \\
\text { cracking }\end{array}$} & & \\
\hline \multirow[t]{2}{*}{9} & \multirow{2}{*}{$\begin{array}{l}\text { Fisura diagonal y de esquina/ } \\
\text { Diagonal and corner crack }\end{array}$} & & & & \\
\hline & & \multirow[t]{2}{*}{23} & \multirow{2}{*}{$\begin{array}{l}\text { Fisuramiento de Bloque/block } \\
\text { cracking }\end{array}$} & & \\
\hline 10 & Losas Subdividas/Sub-divided slabs & & & & \\
\hline 11 & $\begin{array}{l}\text { Deficiencias de material de } \\
\text { sello/Sealing compound failure }\end{array}$ & \multirow[t]{3}{*}{24} & \multirow[t]{3}{*}{$\begin{array}{l}\text { Fisuramiento de Piel de } \\
\text { cocodrilo/crocodile cracking }\end{array}$} & & \\
\hline 12 & Desportillamiento/Chipping & & & & \\
\hline 13 & Levantamiento/Blow-ups & & & & \\
\hline
\end{tabular}

A partir del inventario de daños se establece el Índice de Falla (IF), el cual es calculado con base en el área de la falla y es llevado a términos de porcentaje con respecto al área completa del tramo en estudio (segmento).
From the distress survey, a Distress Index (FI) is established, which is calculated based on distress area and converted into percentages regarding the complete area of the studied (segment). 
Con el porcentaje del área de la falla y su severidad, se ingresa a las curvas de pesos ponderados calibradas para las condiciones de Bogotá. Un ejemplo de las curvas se muestra en la Figura 8. Este modelo de evaluación de fallas está basado en la metodología PAVER desarrollada por Shahin et al., (1990).
By considering the failure area percentage and its severity level, deduct values are calculated from different curves for each failure type. These curves were calibrated for the specific conditions of Bogota city. A curve example is shown in Figure 8. Such evaluation model is based on a PAVER methodology developed by Shahin et al., (1990).

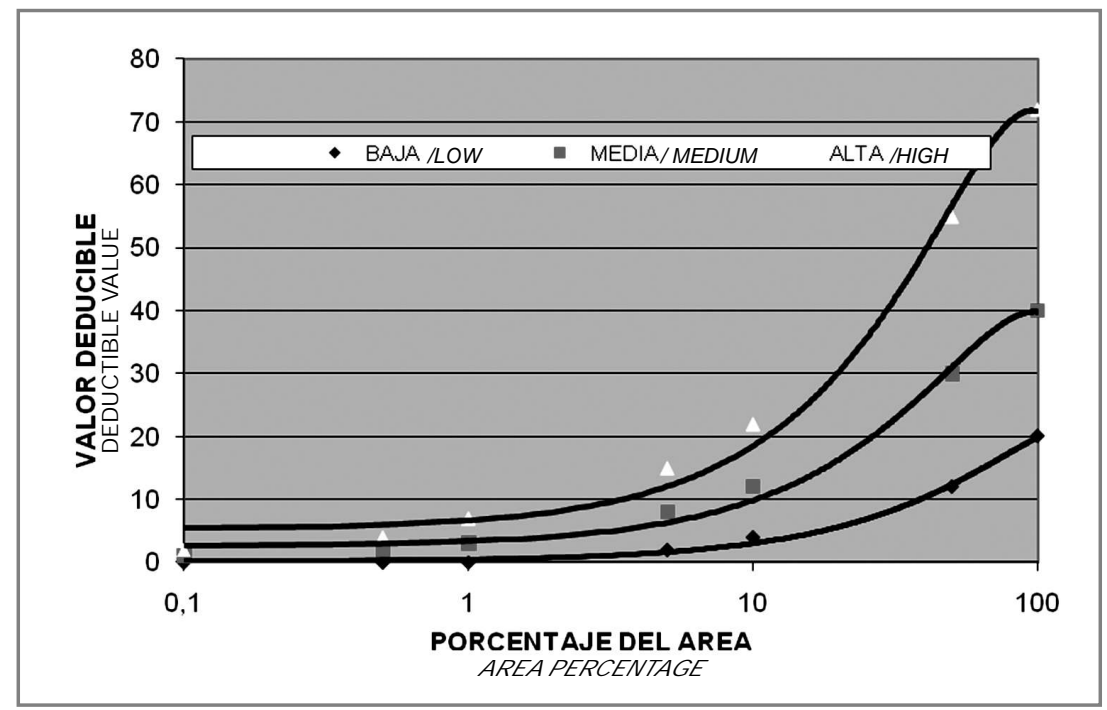

Figura 8. Curvas de pesos ponderados versus área de falla por exudación. (Adaptado de IDU, 2010c) Figure 8. Deduct value v/s bleeding failure area (adapted from IDU, 2010c). (Adapted from IDU, 2010c)

Una vez se cuenta con los pesos ponderados de cada falla (pn) se calcula entonces el IF de acuerdo con la Ecuación 2.
Once distress weighted are obtained from the curve (pn), the index IF can be calculated with the equation 2.

$$
I F=100-\sqrt{p n i^{2}}
$$

Con los resultados del inventario de fallas se obtiene el Índice de Falla (IF) por segmento de ciclorruta, que en conjunto con el IRI permite obtener el Índice de Condición de Ciclorrutas-ICC (IDU (2010c)), empleando la Ecuación 3.
From distress survey results a Distress Index (IF) can be obtained per a bike-path segment, which related with IRI measurements provides a Bike-path Condition Index-ICC (IDU (2010c)), by using the Equation 3.

$$
I C C=\left(\frac{2}{\mathrm{IRI}}\right)^{0.25} \times(84-\mathrm{IF})
$$

El ICC es función del IF y del IRI, obteniendo en un sólo indicador el estado funcional y el estado superficial de la ciclorruta.
ICC is in function of IF and IRI, providing a single indicator for the functional condition and surface condition of the bike-path. 


\section{Revisión y análisis al modelo de gestión existente}

El modelo de gestión vigente se puede observar en la Figura 9. Está compuesto de diferentes módulos: adquisición de datos (inventario), almacenamiento de la información en la base de datos, un módulo de evaluación para la cuantificación del estado del pavimento, un módulo de costos y finalmente un módulo que incluye el universo de intervenciones de mantenimiento.

\section{Analysis and review of existing management model}

The existing management model can be observed in Figure 9. It is composed of different modules: data collection (inventory), information storage in data base, an evaluation model to quantify the pavement condition, a costs modulus and finally a modulus that includes the maintenance intervention alternatives.

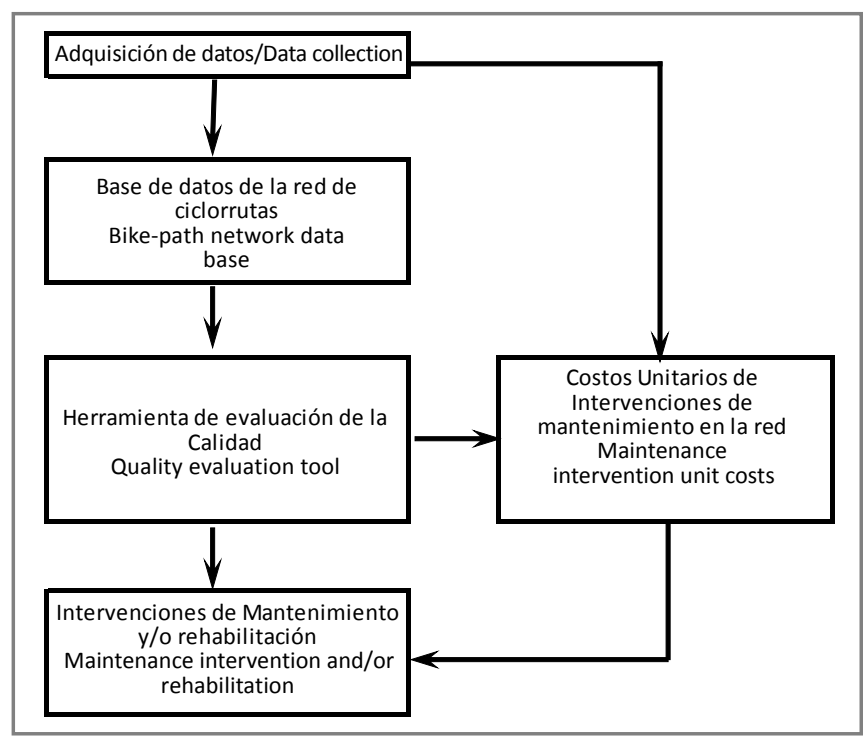

Figura 9. Modelo de gestión existente de la red de ciclorrutas Figura 9. Existing Management Model for bike-path network

\subsection{Revisión del modelo existente}

En consideración a que los listados de falla están basados en la misma metodología llevada a cabo para el inventario de los pavimentos de la malla vial de Bogotá y no obedecen a un estudio realizado para la identificación de daños propiamente en las ciclorrutas, se pueden considerar prácticos, en el sentido de unificar criterios en cuanto a nomenclatura de daños. Pero por otro lado, los listados de fallas son distantes a lo observado en la realidad, en lo que se refiere a los mecanismos de deterioro de una ciclorruta con las distintas clases de superficie de rodadura.

Partiendo del hecho de que la ciclorruta ha sido debidamente construida, a partir de materiales de óptimas calidades y adecuados procesos constructivos, resulta necesario analizar por ejemplo una de las estructuras típicas definidas en el MDC (ver Figura 10). En general, las estructuras están compuestas por un tratamiento superficial con material bituminoso o en la mayoría de los casos por una carpeta de $5 \mathbf{c m}$ de espesor de mezcla densa en caliente.

\subsection{Review of existing model}

Considering that failure lists are based on the same methodology developed for the pavement inventory of Bogota road network and do not correspond to a research conducted to detect failures of bike-path nature, they can be considered as practical reference in order to achieve standard criteria for failure nomenclature. On the other hand, failure lists differ from reality as far as deterioration mechanisms of a bike-path with different type of surface courses are concerned.

Assuming that the bike-path has been properly built from optimum quality materials and adequate constructive processes, it becomes necessary to analyze - for instance - one of the typical structures defined in the MDC (see Figure 10). Generally, the structures used on the bike-path network are composed by a surface treatment with bituminous materials or, in most cases, by a layer of dense warm mixture of $5 \mathrm{~cm}$ thickness. 
El MDC para Bogotá (IDU, 2010b) señala que la estructura de pavimento mencionada debe ser regular, impermeable, antideslizante y de aspecto agradable. Desde la óptica de la conservación de vías cabría la posibilidad de agregar a las características antes mencionadas, el aspecto de la durabilidad.
For Bogota MDC (IDU, 2010b) states that the above mentioned pavement structure must be regular, waterproof, skid-proof and present a good appearance. On the other hand, it would be wise to add durability parameters to above mentioned characteristics.

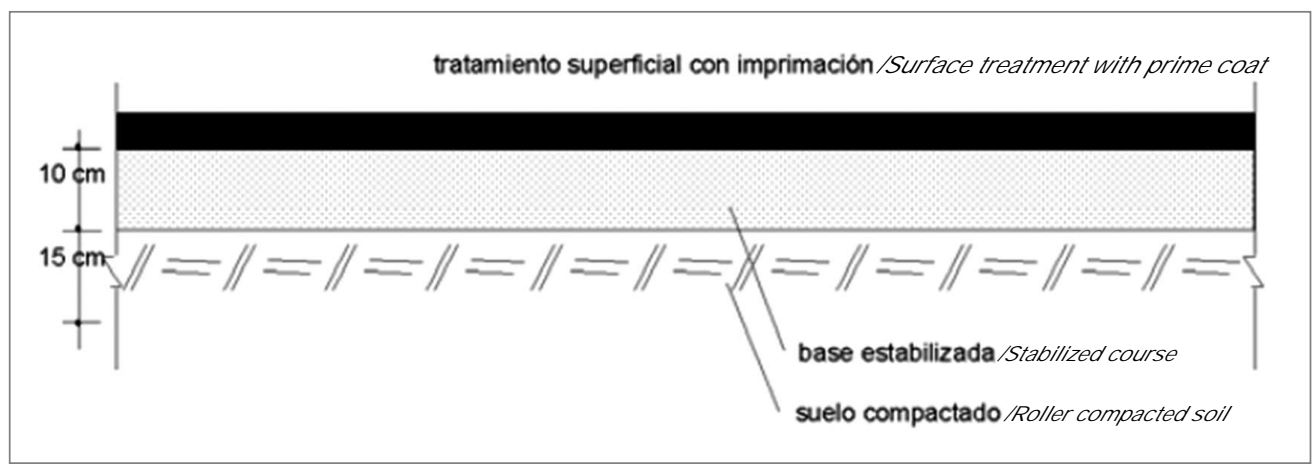

Figura 10. Estructura típica de una cinta de ciclorruta en pavimento flexible. Adaptado de MDC Figure 10. Typical structure of a bike-path belt on flexible pavement. Adapted from MDC

El MDC es claro en señalar que desde el punto de vista estructural, una ciclorruta no es susceptible de grandes esfuerzos, por lo tanto es trivial descartar dentro del listado de daños, aquellos originados por esfuerzos repetitivos, como pueden ser: desplazamientos, hundimientos y fisuración tipo piel de cocodrilo (asociada con fatiga). No obstante es posible observar este tipo de fallas, ya que éstas pueden ser relacionadas con malos procesos constructivos, materiales de mala calidad y efectos de agentes externos a la ciclorruta. En las Figuras 11 a la 13 se observan algunas de las fallas más presentadas en la red de ciclorrutas y que no están consideras dentro del listado de fallas existente.
MDC clearly indicates that, from a structural point of view, a bike-path is not susceptible to undergo high levels of stress, therefore one could eliminate from the failure list those originated by repetitive stresses, such as: displacements, settlements and crocodile cracking (associated to fatigue damage). However, it is possible to observe such type of failures since they can be originated by poor constructive processes, poor quality materials and bike-path network external agent effects.

From Figures 11 to 13, some of the most common failures are shown; however, they are not included in the existing failure list for bike-path network.

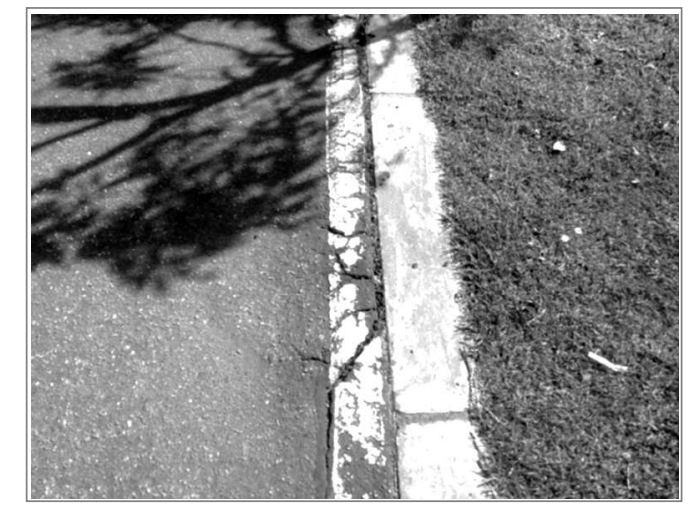

Figura 11. Fisuras concentradas sobre la línea de demarcación

Figure 11. Cracking concentrated on demarcation line 


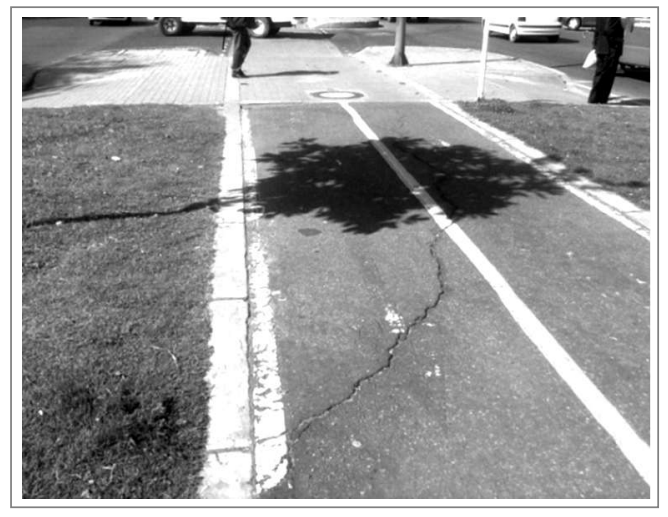

Figura 12. Fisura en diagonal causada por expansión de Subrasante Figure 12. Crossover cracking caused by sub-grade line expansion

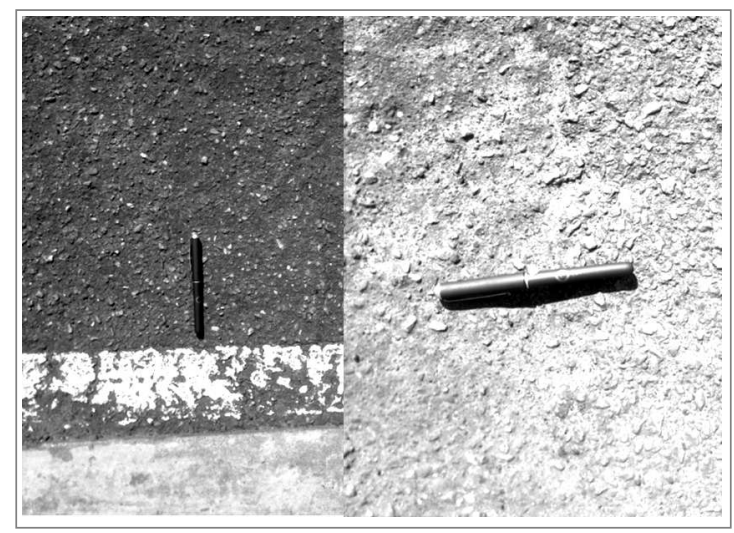

Figura 13. Desgaste superficial en ciclorrutas, originado por efecto climático Figure 13. Raveling on bike-paths caused by climatic changes

\subsection{Mecanismos de deterioro en las ciclorrutas}

De acuerdo con World Bank (1988) se definen como determinantes del deterioro de un pavimento los siguientes aspectos:

a. Topografía y subrasante

b. Material y espesor de la capa de pavimento

c. Drenaje (superficial y externo)

d. Calidad de la construcción y del mantenimiento

e. Medio ambiente (lluvia, congelamiento, radiación solar)

f. Tráfico

g. Condición del Pavimento
5.2 Deterioration mechanisms for bike-paths According to the World Bank (1988), the following aspects are defined as key factors for pavement deterioration:

a. Topography and sub-grade line

b. Material and thickness of pavement layer

c. Drainage (surface and external)

d. Construction and maintenance quality

e. Environmental conditions (rainfall, freezing and solar radiation)

f. Traffic

g. Pavement conditions 
Para la estructura en análisis (ver Figura 10), partiendo del hecho de que la estructura se encuentra debidamente construida, cobran especial importancia los deterioros asociados con el medio ambiente, siendo la causa preponderante en la gran de red de ciclorrutas de Bogotá (ver Figura 13). Revisando el estado del conocimiento en el área de envejecimiento foto-químico de mezclas asfálticas y por efecto ambiental, se encuentran trabajos de Tia et al., (1988), en donde el efecto de la radiación ultravioleta lo limitan a los primeros milímetros. Hugo et al. (1985), encuentran efectos de endurecimiento dentro de los primeros $5 \mathrm{~mm}$ y los comparan con viscosidades de $\mathbf{2} \mathbf{~ m m}$ más profundas en donde ya no se observa ningún efecto por la radiación. En el trabajo de Kempt et al. (1981), se afirma que la radiación con luz "actínica" afecta hasta $5 \mu \mathrm{m}$ de la película de asfalto. En este orden de ideas queda evidenciado que no existe consenso en cuanto a la profundidad de afectación, teniendo en cuenta que los trabajos reportados han sometido distintas clases de mezclas en estados semicompactos y periodos de exposición diferentes.

En el contexto de los asfaltos colombianos se han reportado trabajos realizados en el área de envejecimiento de mezclas asfálticas por Martínez et al., 2005, quienes mediante el estudio de una mezcla asfáltica densa con asfalto 80-100 de penetración, utilizando técnicas de envejecimiento acelerado en un Weatherómetro $U V$, determinaron que la radiación ultravioleta alcanza a afectar hasta una profundidad de $15 \mathrm{~mm}$. Para el caso de las ciclorrutas, una afectación por envejecimiento de $15 \mathrm{~mm}$ es el equivalente al $\mathbf{3 0} \%$ del espesor total $(50 \mathrm{~mm})$, por lo tanto debe considerarse como un efecto notable en la vida útil de la rodadura. Posibles actividades de mantenimiento involucrarían tratamientos con agentes rejuvenecedores o tratamientos superficiales tipo slurry o micro pavimentos. Por otro lado, sería posible evaluar la implementación de asfaltos modificados en la construcción de ciclorutas, los cuales han demostrado generar una mayor película de recubrimiento al agregado por su mayor viscosidad en relación con un cemento asfáltico convencional, y por otro lado, una mayor resistencia al envejecimiento (Airey et al., 2002, Elseifi et al., 2003, Airey 2004, Martínez et al., 2006).

La tendencia mostrada de las ciclorrutas a presentar desgaste o deterioro superficial, motiva a proponer medidas que evalúen de forma cuantitativa el estado superficial de la rodadura y el confort percibido por los usuarios.
For the structure under analysis (see Figure 10), assuming this structure was properly constructed, deterioration associated to environmental changes become especially relevant as it is the predominant factor affecting the bike-path network in Bogota (see Figure 13). By checking up the acquaintance state of photo-chemical ageing on asphaltic mixtures and due to environmental changes, researches conducted by Tia et al, (1988) are found, who states that ultraviolet radiation limits ageing only to depth-first millimeters. Hugo et al. (1985) found hardening effects in depth-first $5 \mathrm{~mm}$ and compared them with $\mathbf{2 m m}$ even deeper viscosities where no effect is observed due to radiation. Kempt et al. (1981) stated that radiation from "actinic" light affects up to $5 \mu \mathrm{m}$ of asphalt layer. In this sense, it becomes evident that there is no agreement regarding affection depth, taking into account that reported studies have evaluated different types of mixtures under semi-compacted conditions and different exposure periods.

In the context of Colombian asphalts, an investigation on asphaltic mixtures aging have been conducted by Martinez et al, 2005, who by means of the study on a dense asphaltic mixture with 80-100 penetration grade asphalt, and employing accelerated aging techniques in a UV weather meter, determined that ultra-violet radiation affects only up to a $15 \mathrm{~mm}$ depth. In the case of bike-paths, the aging affectation of $15 \mathrm{~mm}$ is equivalent to $30 \%$ of total thickness $(50 \mathrm{~mm})$, therefore it shall be considered as a remarkable effect on a course operational life time. Potential maintenance activities would involve surface treatments using anti-aging agents or slurry-micro surfacing treatments. On the other hand, it would be possible to evaluate the implementation of modified asphalts for bike-path constructions, which have proved to provide a thicker coating layer on aggregates due to a higher viscosity compared to a conventional asphaltic cement mixture, and also deliver higher anti-ageing strength (Airey et al., 2002; Elseifi et al., 2003; Airey 2004; Martínez et al., 2006).

Bike-paths are prone to undergo surface wearingout and deterioration which encourage the achievement of measures providing a quantitative evaluation on the course surface condition and comfort perceived by users. 
Actualmente, se determina el estado superficial por desgaste (peladuras) mediante inspección visual (IDU (2010c)), proceso que incluye un alto porcentaje de subjetividad por parte del inspector y escasa repetitividad en los inventarios. Para contar con una medida directa sobre el estado de desgaste o pérdida de ligante en la rodadura asfáltica, podría ser de gran ayuda realizar mediciones de textura (macrotextura) a través de métodos estáticos, tales como el círculo o parche de arena, el medidor de flujo (outflow meter) y el medidor de textura circular (CTMeter). De estos métodos, se destaca el método del parche de arena como un estándar por su amplia aceptación y difusión y la gran cantidad de datos históricos existentes (Flintsch et al., 2005). La evaluación y monitoreo de la macrotextura de la superficie garantizara la seguridad operacional en las ciclorrutas, ya que este parámetro condiciona la resistencia al deslizamiento de superficies en condiciones húmedas (NCHRP, 2009).

Por otro lado, el Índice de Condición de Ciclorrutas-ICC (Ecuación 3) está condicionado a la evaluación del Índice de Rugosidad Internacional (IRI) en el tramo bajo estudio. El IRI es un parámetro utilizado para evaluar la calidad de tramos de vía, el cual está fundamentado en la respuesta dinámica de un modelo vibracional que simula un cuarto de carro estándar viajando a una velocidad de $80 \mathrm{~km} / \mathrm{h}$ sobre la superficie evaluada. El modelo original del IRI es presentado en la Figura 14.
Currently, the surface condition due to wearing-out (scabbing) is determined by means of a visual inspection (IDU 2010c)). Such process is highly subjective and scarcely repetitive for inventories. In order to evaluate wearing conditions or binder loss in the asphaltic course directly, one must carry out texture measurements on pavement, by means of static methods, such as the sand circle or sand patch methods, outflow mete, circular texture meter (CT meter) are recommended. The sand patch stands out from such methods as a widely accepted and broadcasted standard, due to high amount of historical data available (Flintsch et al, 2005). The evaluation and monitoring on surface macrotexture will guarantee safe operational conditions on bike-paths, since this parameter determines skid resistance on wet condition surfaces (NCHRP, 2009).

On the other hand, the Bike-path Condition Index-ICC (Equation 3) is conditioned to the evaluation of International Roughness Index (IRI) for the segment under study. IRI is a parameter employed to evaluate the quality of bike-path segments, and it is based on the dynamic response from a vibration model simulating a standard quarter-car traveling at $80 \mathrm{~km} / \mathrm{h}$ on the evaluated surface. The original IRI model is shown in Figure 14.

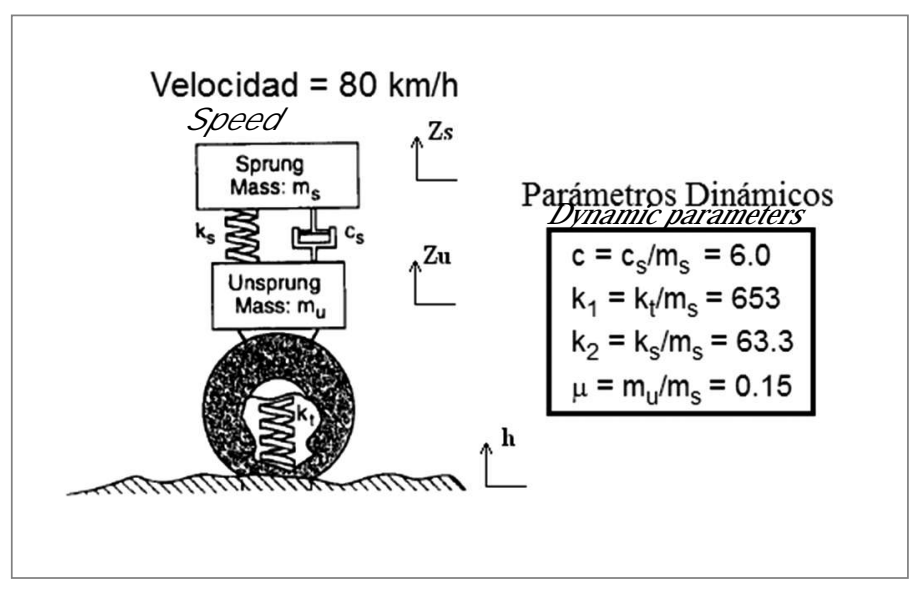

Figura 14. Modelo de un cuanto de carro utilizado para el cálculo del IRI (Modificado de Sayers, 1995) Figure 14. A quarter-car used for IRI calculation (Modified from Sayers 1995) 
Basados en estos conceptos podemos esperar que la respuesta dinámica percibida por un ciclista al transitar por una superficie sea muy distinta a la representada por el modelo del IRI, ya que el sistema de suspensión y los diferentes parámetros dinámicos de estos dispositivos (Vehículo - Cicla) difieren significativamente. Por otro lado, la velocidad promedio de un ciclista es significativamente menor (estimada entre $15-20 \mathrm{~km} / \mathrm{h}$ ) a la velocidad utilizada por el modelo del IRI. Esto resulta significativo ya que la respuesta dinámica de un vehículo a transitar por una superficie es altamente sensible a la velocidad de viaje de este (Fuentes et al 2010)

Bajo este contexto, resulta pertinente la reevaluación del IRI como parámetro de control de la calidad de las ciclorrutas, ya que este carece de significado y no representa el nivel de confort experimentado por un ciclista a recorrer un tramo de vía. El modelo recalibrado del IRI deberá representar la respuesta dinámica de una cicla al transitar por una tramo de via a una velocidad operacional representativa de estos dispositivos.

En consideración a lo anterior, se observa como un elemento clave para la efectividad del Sistema de Gestión de Ciclorrutas (SGC) el entendimiento del deterioro de las ciclorrutas ocasionado por los efectos ambientales, como la fatiga térmica en las rodaduras asfálticas y el endurecimiento por efecto fotoquímico. El conocimiento en profundidad de las causas del deterioro de los pavimentos permitirá la inclusión de estrategias y procesos constructivos dirigidos a contrarrestar los mecanismos de deterioro identificados. Con el objetivo de planificar y estimar el desempeño de la red, se requiere de manera primordial la definición de tramos testigos que permitan desarrollar ecuaciones o curvas de deterioro y faciliten la estimación del desempeño de las ciclorrutas, brindando un soporte importante para la planeación de inversiones en el tiempo, optimizando los recursos del gobierno distrital y conservando la red de ciclorrutas en condiciones de servicio concordantes a los estándares definidos como parte la política de la autoridad en infraestructura.

\section{Aspectos que se deben implementar al Sistema de Gestión existente}

Como resultado de la revisión al SGC existente, se pudieron determinar falencias que se describen en los numerales 6.1 al 6.5 .
Based on these concepts it is expected that dynamic response sensed by a biker riding on a surface would be quite different from the one represented by the IRI model, since suspension system and different dynamic parameters of these devices (vehicle - bike) are significantly different. On the other hand the average speed of a bike (estimated in $15-20 \mathrm{~km} / \mathrm{h}$ ) is remarkably lower than the speed employed by IRI model. This represent a significant fact since the dynamic response of a vehicle running on a surface is highly sensitive to its speed (Fuentes et al 2010).

In this context, it is advisable to re-evaluate IRI as a control parameter on bike-paths quality conditions, since it lacks of meaning and does not represent the comfort level experienced by a biker when riding on a bike-path. Re-calibrated IRI model should represent the dynamic response for a bicycle riding on a bike segment at an operational speed representative for such devices.

Considering the above, a key element for the effectiveness of a Bike-path Management System (SGC) is the understanding of bike-path deterioration due to environmental changes, such as thermal fatigue on asphaltic courses and hardening due to photo-chemical effect. A deep knowledge of pavement deterioration will allow the implementation of strategies and construction processes intended to reduce the identified deterioration mechanisms. For the planning and assessment of the bikepath network performance, a definition of witness sections is fundamentally required to develop equations and deterioration curves facilitating the evaluation of bikepaths performance, thus providing a significant support for investment plans throughout time, leading to the optimization of district government resources and to the conservation of bike-paths under service conditions fulfilling the standards defined as integral part of district infrastructure policies.

\section{Aspects to be implemented to the existing Management System}

As a result from the review of existing SCG, weaknesses of the current system were determined and are described from 6.1 to 6.5 . 


\subsection{Información para los inventarios}

La información record de las ciclorrutas registrada una vez se finaliza su construcción, así como, la información levantada en campo y consignada en los inventarios, no es suficiente para poder realizar análisis del sistema en cuanto a las causas del deterioro y desempeño de la red, debido a que en la base de datos no se cuenta con la información referente a los espesores de las capas de las ciclorrutas y a la calidad de los materiales empleados. Una de las causas de las fallas encontradas de manera reiterativa en la red, es la fisuración y pérdida de la regularidad de la ciclorruta por efecto de las raíces de los árboles, información que se considera necesaria incluir en los inventarios de la ciclorrutas. Posibles soluciones a esta patología requerirán una especificación en cuanto a la distancia mínima entre el eje de las ciclorrutas y la presencia de árboles en sus vecindades.

Para complementar la información que actualmente se consigna en un inventario de la red de ciclorrutas, se propone el listado de variables que se presenta en la Tabla 3.

\subsection{Information for Inventories}

Record information on bike-paths network registered after construction is completed, as well as the information obtained from field surveys for inventory purposes, is not enough to conduct a system analysis regarding deterioration causes and network performance. Since data base does not have information on the bikepath layer thicknesses neither on employed material quality. One failure cause repetitively found is cracking and loss of surface evenness in bike-path network due to trees roots, hence this information must be included in bike-paths inventories. Possible solutions to such pathology will demand a specification regarding the minimum free distance from bike-paths center axle to trees present in the neighborhoods.

In order to complement the existing information on bike-paths inventories, the following variable listing is proposed, as shown in Table 3.

Tabla 3. Datos que se deben incluir en el inventario de ciclorrutas

Table 3. Data to be included in bike-path inventories

Característica/Characteristics
Tipo de superficie/Type of surface
Ancho de superficie/Surface width
Tipo de sección (e.g. sobre andén, separador, contiguo a la vía)/Type of section (e.g. on platform, spacer, adjoining the lane)
Zona aledaña ( e.g. impermeable ó permeable)/Bordering zone (waterproof or permeable)
Espesor de capas (e.g. todas las capas sobre la subrasante)/Layer surface thickness (all layers over sub-grade line)
Espaciamiento de juntas (en pavimento rígido)/Joints spacing (rigid pavement)
Tipo de Subrasante y clasificación (e.g. sistema AASTHO, USCS*)/Type and classification of sub-grade line (e.g. AASTHO, USCS* system)
Presencia de arboles en la vecindad (tipo de especie, distancia)/Presence of trees in the neighborhood (species type, distance)
Pendiente longitudinal/Slope Length
Índice de estado ciclorruta/Bike-path Condition Index
Índice de estado de la demarcación/Boundary marking condition index
Volumen de usuarios en bicicletas/Bike-path user volume
Nivel socio-económico del sector/Socio-economical level in the zone
*United Soil Classification System/United Soil Classification System
* American Association of State Highway and Transportation Officials/American Association of State Highway and Transportation Officials

6.2 Modelos de comportamiento

Para poder estimar el desempeño y deterioro de las ciclorrutas, es primordial contar con tramos testigos a los cuales se les realice un seguimiento periódico sobre su estado y velocidad de deterioro. Esta información facilitará la formulación de modelos de deterioro para cada una de las familias de segmentos analizados y proveerán al IDU de herramientas empíricas para la estimación de vida útil y tiempo óptimo para adelantar labores de mantenimiento (George, 2000, Khattak et al, 2008).

\subsection{Behavior Models}

In order to assess bike-paths performance and deterioration, it is essential to define witness sections, which shall be periodically monitored to evaluate the conditions and deterioration speed. Such information will be useful for the development of deterioration models for each segment group under study and they will provide IDU with empirical tools for assessing life time and optimal periods to perform maintenance tasks in advance (George, 2000; Khattak et al,, 2008). 


\subsection{Estándares de conservación}

Los estándares de conservación manifiestan los umbrales de precaución para las agencias, considerando como el momento o estado en el cual un determinado segmento de la red requiere alguna clase de actividad de mantenimiento para garantizar la serviciabilidad del tramo. Para la red de ciclorrutas no se conocen, ni se han establecido umbrales de prevención, como se discutió en 5.2, además de indicadores en términos de porcentaje de área fallada y rugosidad. Podría ser de gran ayuda contar con un estándar de conservación dirigido a evaluar el desgaste por efecto del clima.

\subsection{Evaluación Social}

La evaluación social debe ser uno de los elementos centrales para la toma de decisión para efectos de priorizar intervenciones, y evaluar el impacto de las mismas en la población beneficiada al momento de realizar una intervención sobre la red de ciclorrutas (Chamorro et al., 2009). Es claro que la bicicleta en el contexto de Bogotá no es valorada de la misma manera en todos los estratos económicos, representando en los estratos bajos un medio de transporte invaluable comparable con cualquier sistema de transporte de la ciudad (IDU 2010b). Por lo anterior resulta incuestionable que todo proceso de toma de decisiones valore el número de usuarios de la ciclorruta en una zona determinada y la clase de uso que le dan los mismos al sistema (medio de transporte, recreativa etc.).

\subsection{Herramienta para toma de decisiones}

Una herramienta sistemática para la toma de decisiones será la que conglomerará todas las variables y datos de entrada del sistema, para que mediante el empleo de técnicas de optimización muestre los reportes de prioridad y tipo de intervenciones para garantizar la serviciabilidad deseada acorde con las políticas de mantenimiento establecidas por la agencia competente. El modelo de gestión existente con los elementos que deberían ser incluidos al sistema se muestra en la Figura 15.

\subsection{Conservation standards}

Conservations standards provide agencies with precautionary thresholds, alerting the moment or condition when a given network segment requires some kind of maintenance action, thus guaranteeing such zone serviceability. There is no information whether precautionary thresholds have been implemented for bike-path networks, as discussed on 5.2, neither on indicators for failure area percentages or roughness indicators. Hence, a conservation standard intended to evaluate deterioration due to climate changes would be a great contribution.

\subsection{Social Assessment}

Social assessment is a key element to be considered at the stage of prioritizing interventions and their impact on target community in a bike-path network. (Chamorro et al 2009). It is quite clear that in Bogota, bicycles are not valued in the same manner by all economical stratums, being is an invaluable transportation means for lower class when compared to any other transportation system available in the city (IDU 2010b). Therefore, it becomes unquestionable that all decisionmaking process shall consider the bike path number of users for a given zone and the kind of use they require from the system (transportation, recreation means, etc.)

\subsection{Decision Making Tool}

A systematic decision making tool shall bring all variables and system data collected together, so that, by means of the use of optimization techniques, it shall provide priority reports and the type of interventions required to guarantee desired serviceability in accordance to maintenance policies established by the competent authority. The existing management model and the elements to be included in the system are shown in Figure 15. 


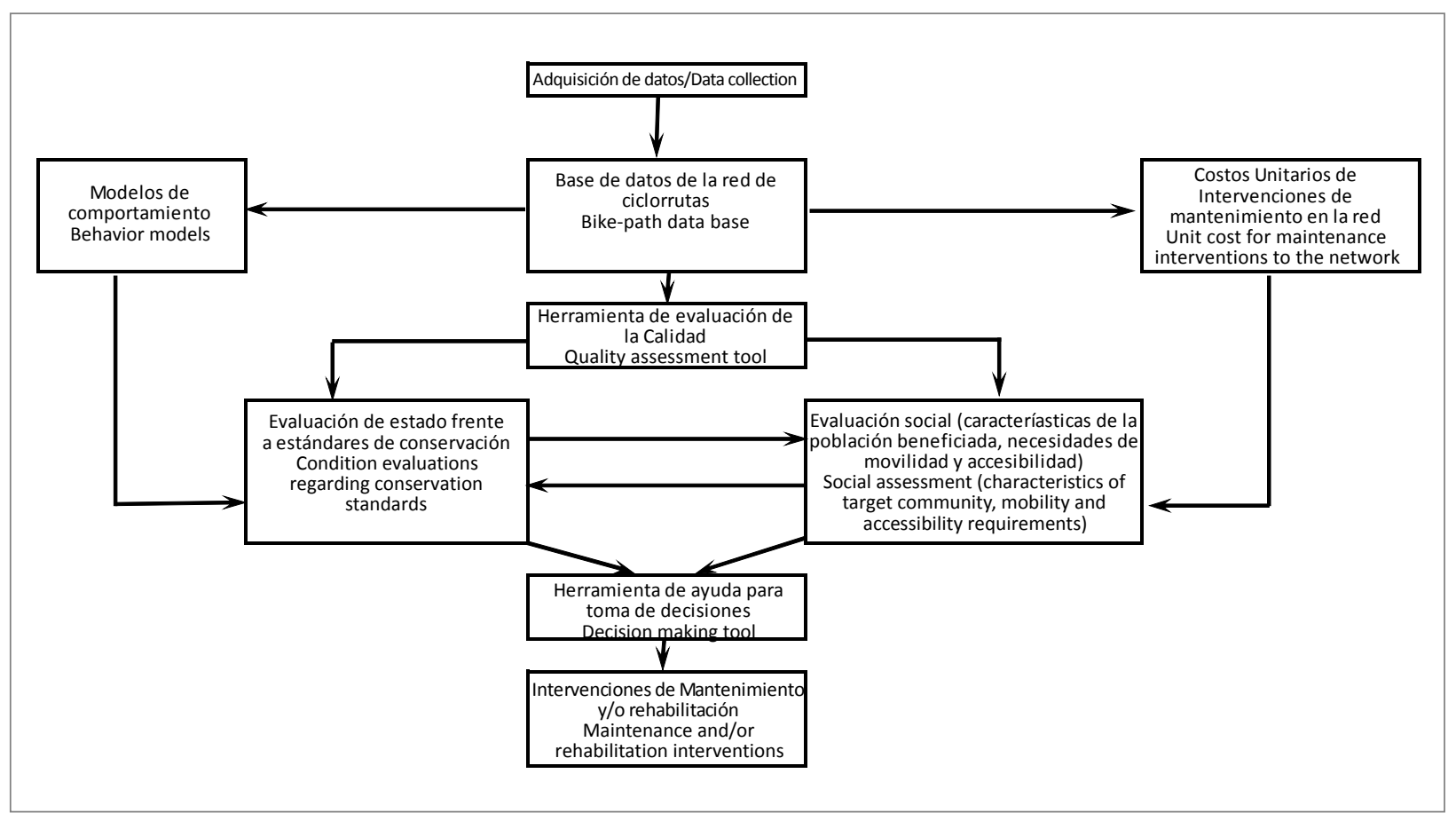

Figura 15. Modelo de Gestión existente con la incorporación de los elementos propuestos con base en las falencias detectadas Figura 15. Existing Management System considering proposed elements based on failures detected

\section{Conclusiones}

El artículo ha revisado de manera integral el sistema de gestión de ciclorrutas existente para la ciudad de Bogotá. Como conclusiones del trabajo se encontraron:

a. Se hace necesario el establecimiento de un manual de fallas para ciclorrutas que contenga los posibles deterioros en este tipo de estructuras. Identificar los tipos de daños en ciclorrutas, con base en un manual para daños de vías vehiculares, carece de objetividad y exactitud, por cuanto el modo, origen y nivel de deterioro es diferente.

b. El sistema actual no cuenta con modelos de deterioros o herramientas para este fin. La predicción del deterioro de las ciclorrutas requiere de la definición de tramos testigos que alimenten una base de datos que permita el desarrollo de modelos empíricos para la estimación y predicción de los deterioros en las ciclorrutas.

c. El índice de condición de ciclorrutas está basado en el IF y en el IRI, ambos parámetros desarrollados para vías vehiculares. Se hace necesario estudiar un Índice de Condición que involucre parámetros directamente relacionados con el confort del usuario no-motorizado, posiblemente algún parámetro relacionado con textura.

\section{Conclusions}

This paper has integrally reviewed the existing bike-path management system of Bogota city. The following conclusions were achieved:

a. It is necessary to develop a bike-path failures guideline containing all potential deterioration elements for this kind of structure. Identifying the kind of failures in bike-paths based on a vehicular road guideline is worthless, as it lacks of objectivity, taking into account that origin of the failure and the deterioration levels are quite different.

b. The current system does not include deterioration models or tools required for this purpose. Bike-path deterioration prediction requires the definition of witness sections to feed a data base allowing the development of empiric models for the assessment and prediction of bike-paths deterioration.

c. The bike-path condition index is based on IF and IRI, both parameters created for vehicle roads. It is necessary to develop a Condition Index directly involving parameters related to the comfort of nonmotor vehicle users, perhaps including a texture parameter. 
d. Para el modelo de gestión existente se proponen elementos que complementarán el actual sistema, y de esta manera el sistema cuente con los elementos mínimos que debe llevar un sistema de gestión de infraestructura, tales como el desarrollo de modelos de deterioro, definición de estándares de mantenimiento y la evaluación social en la fase de priorización de intervenciones.

e. Con base en la información existente y en la inspección realizada a la red de ciclorrutas se evidenció que el desgaste superficial en las ciclorrutas con rodadura bituminosa originado por efecto climático, debe ser considerado como una falla de efecto significativo, que obliga considerar nuevas técnica constructivas empleando quizás asfaltos modificados, los cuales presentan una mayor resistencia al envejecimiento por intemperismo

f. Los resultados mostrados en el presente artículo, propenderán en la adecuada y oportuna intervención en las labores de conservación de las ciclorrutas, garantizando la inversión de los recursos en los tramos priorizados por el modelo de predicción y por el sistema de administración de las ciclorrutas. d. Complementary elements are proposed to the existing management system to achieve a system counting with the minimum elements considered by an infrastructure management system, such as development of deterioration models, definition of maintenance standards and social assessment during intervention prioritizing stages.

e. Based on the existing information and on the review conducted on bike-path network, surface wearing out on bituminous course mainly due to climate changes became evident as one of the distinctive deterioration mechanism. Therefore one should consider new constructive techniques by using modified asphalts, which deliver higher anti-aging resistance against weathering effects.

f. The results shown in this paper will lead to an adequate and timely intervention of conservation tasks on bike-paths, thus guaranteeing resources investment on segments prioritized by prediction model and by bike-path administration system.

\section{Referencias / References}

Airey G. 2004, "Fundamental binder and practical mixture evaluation of polymer modified bituminous materials". The International Journal of Pavement Engineering, Vol 5(3), p 137-151.

Airey G. D., Singleton T. M. y Collop A. C. (2002), "Properties of polymer modified bitumen after rubber-bitumen interaction". Journal of Material in Civil Engineering", Vol 14(4) August 1, p 344-354.

Chamorro A. y Tighe S. L. (2009), Development of a management framework for rural roads in developing countries. Journal of the Transportation Research Board of the National Academies, Washington, DC.

De Solminihac H. (2005), Gestión de Infraestructura Vial, Tercera edición. Editorial Alfaomega Grupo Editor, Colombia.

Elseifi M. A., Flintsch G. W. y Al-Qadi I. L. (2003), “Quantitative Effect of Elastomeric Modification on Binder Performance at intermediate and High Temperature", Journal of Materials in Civil Engineering, Vol. 15, No 1, p. 32-40.

Flintsch G., De León E. y Medina A. (2005), La macrotextura superficial del pavimento como indicador de calidad en pavimentos flexibles (CD). XIII Congreso Iberolatinoamericano del Asfalto, San José, Costa Rica.

Fuentes L., Gunaratne M. y Hess D. (2010), Evaluation of the Effect of Pavement Roughness on Skid Resistance, ASCE Journal of Transportation Engineering. Volume 136, No 7, pp. 640-653

George K. P. (2000), “MDOT Pavement Management Systems, Prediction models and feedback systems". Department of Civil Engineering The University of Mississippi. Report FHWA/MS-DOT-RD-00-119

Gharaibeh N., Wilson C., Darter M. y Jones G. (1998), Development of a bike path Management System for the University of Illinois at Urbana-Champaign. Tranportation Research Record No. 1636 Bicycle and Pedestrian Research 1998. Paper No. 98 -0331

Haas R. W, R. Hudson y J. P. Zaniewski (1994), Modern Pavement Management. Krieger Publishing Company. Malabar, Florida.

Hugo, F. and Kennedy, T.W. (1985), Surface cracking of asphalt mixtures in Southern Africa, Proc. Assn. Asphalt Paving Technol. 54, 454-501.

Instituto de Desarrollo Urbano de Bogotá (2010a), http://www.idu.gov.co/web/guest/espacio_ciclorutas. Fecha de última visita: Marzo 2010.

Instituto de Desarrollo Urbano de Bogotá (2010b), Plan Maestro de Ciclorrutas. Manual de Diseño de ciclorrutas. http://www.idu.gov.co/web/guest/espacio_ciclo_plan. Fecha de última visita: Marzo 2010. 
Instituto de Desarrollo Urbano de Bogotá (2010c), Plan Maestro de Sostenibilidad de la Infraestructura Urbana de Bogotá. http://www.idu.gov.co/web/guest/tramites_doc_planmaestro. Fecha de última visita: Marzo 2010.

Instituto de Desarrollo Urbano de Bogotá (1999), TNM Limited. Plan Maestro de Sostenibilidad Vial IDU - TNM contrato 834 de 1999.

Kemp G.R. y ProdoehI N. H. (1981), A comparison of field and laboratory environments on asphalt durability2, Proc. Assn. Asphalt Paving technol. 50. 492-537.

Khattak M. J., Baladi G. Y., Zhang Z. y Ismail S. (2008), Review of Louisiana's Pavement Management System. Transportation Research Record: Journal of the Transportation Research Board, No. 2084, Transportation Research Board of the National Academies, Washinton, D.C., pp. 18-27.

Martínez G. y Caicedo B. (2005), Efecto de la radiación ultravioleta en el envejecimiento de ligantes y mezclas asfálticas. Congreso Ibero-Latinoamericano del Asfalto CILA XIII, San José de Costa Rica.

Martínez G., Caicedo B., Gonzáles D. y Celis L. (2006), Rheological Behaviour of Asphalt with Crumbed Rubber and other Modifiers. Asphalt Rubber Conference 2006. Palm Springs.

NCHRP (2009), Guide for Pavement Friction Project No. 01-43. Transportation Research Board. National Research Council. Sayers M. (1995), On the Calculation of International Roughness Index from Longitudinal Road Profile, Transportation Research Record 1501, Transportation Research Board Business Office, Washington, D.C., pp. 1-12.

Shahin M. Y. y Walter J. A. (1990), "Pavement Maintenance Management for Roads and Streets Using the PAVER System". U.S Army Corps of Engineers (USA CERL), Champaign II, Technical Report M-90/05.

World Bank. (2000), Highway Development and Management Series. Volumen 1. Visión General del HDM-4, versión Piarc R101, París. World Bank. (1988), Road deterioration in developing countries - causes and remedies. Washington. 